\title{
Evaluación de la producción y calidad de huevos en pollas ponedoras (Loman brown) en el Centro Multipropósito Monte Las Palmas, Chocó, Colombia
}

\section{Evaluation of the production and quality of eggs in laying hen (Loman brown) in Center Multipurpose Monte Las Palmas, Choco, Colombia}

\begin{abstract}
Ariel Castro*, Leider Palacios*, Sandra María Gutiérrez*, Yohana Mendoza*
Mediante el establecimiento, seguimiento y manejo de registros de producción en dos lotes con 400 pollas ponedoras cada uno, se evaluó la producción y calidad de huevos en pollas ponedoras Loman brown, en el Centro Multipropósito Monte las Palmas, en el departamento del Chocó. Se utilizó como fuente de alimentación $100 \mathrm{~g}$ de concentrado ave/día, los pastos imperial (Axonopus micay, Axonopus scoparius), y maralfalfa (Pennisetum sp.) a manera de ensayo. Se logró en buena medida que los animales pudieran manifestar su potencial genético en un ambiente controlado técnicamente, disminuyendo costos en su producción, pues la calidad de los huevos se determinó por el tipo de alimentación suministrada; se destaca la utilización de los pastos, que influye de manera significativa en la convivencia de las pollas, el mejoramiento en la textura externa, el tamaño de los huevos y la calidad de la yema.
\end{abstract}

Palabras clave: Chocó; Loman brown; Pasto imperial; Pasto maralfalfa.
* Grupo de Investigación en Actividad Farmacológica «DARIÉN», Universidad Tecnológica del Chocó «Diego Luis Córdoba», Quidbó, Colombia.

e-mail: aricasbel09@hotmail.com leipal@hotmail.com samtie@hotmail.com yohanitamen@yahoo.com

Recibido: 17 de marzo de 2010 Aceptado: 7 de abril de 2010

\section{ABSTRACT}

By means of the establishment, pursuit and handling of registries of production in two lots with 400 laying pullets each, evaluated the production and quality of eggs in laying pullets Loman brown, en the Centro Multipropósito Monte las Palmas, in the department Choco. Bird was used like power supply $100 \mathrm{~g}$ of concentratelday and the grass Imperial (Axonopus micay, Axonopus scoparius), and Maralfalfa (Pennisetum sp.) voluntarily. It was obtained largely that the animal could indicate their genetic potential in a controlled environment technically, diminishing costs in his production, since the quality of eggs was determined by the type of provided feeding the pullets; the use of the grass stands out, that influences significantly in the coexistence of the pullets, improvement in the external texture and the size of eggs and the quality of the yolk.

Keywords: Chocó; Loman brown; Grass imperial; Grass maralfalfa.

\section{INTRODUCCIÓN}

El objetivo de todo avicultor es obtener buenos ingresos de su explotación. Para ello su negocio debe ser rentable y sufragar, de manera parcial o total los gastos de su familia, amortizar inversiones y obtener una ganancia. La rentabilidad se determina por factores externos, que no puede controlar directa el avicultor, tales como el alza en los precios de los alimentos concentrados y otros insumos, o baja en precios de venta de los productos. Con una buena administración se pueden reducir los riesgos que presentan esos factores externos, así, la explotación puede ser rentable mediante una buena planificación, organización, control y dirección de los procesos productivos. Estos procesos a su vez pueden ser 


\section{Bioetnia Volumen 7 № 1 (enero-junio), 2010}

técnicos o financieros, como conversión alimenticia, mortalidad, tensión o estrés, etc., o malas inversiones que afecten el flujo de caja (Bonino 1994). En el departamento del Chocó, el estudio y producción de animales avícolas es incipiente; por tradición se recurre a prácticas de cría de pollas de tipo local para el consumo familiar; sin embargo, la mayoría de los productos que ofrece este recurso es introducido por pequeños comerciantes que desarrollan sistemas de producción animal a gran escala para uso extensivo de comercialización y distribución en la región.

El objetivo de esta investigación fue evaluar la producción y calidad de huevos en pollas ponedoras raza Loman brown, en el Centro Multipropósito Monte las Palmas, utilizando como fuente de alimentación dos tipos de pastos (imperial y maralfalfa), mediante el establecimiento, seguimiento y manejo de registros de producción en dos lotes, para lograr en buena medida que los animales puedan manifestar todo su potencial genético en un ambiente técnicamente controlado, disminuir costos en su producción y aumentar la rentabilidad económica en las familias chocoanas.

\section{GENERALIDADES DE LOS FORRAJES}

Según Bernal etal. (1997), Rua(2008)y Romero(2007), los pastos de corte, en especial el imperial (Axonopus micay, Axonopus scoparius) y maralfalfa (Pennisetum sp.), se adaptan con gran versatilidad a pisos térmicos entre los 0 y 1800 metros de altura, algunos de ellos pierden productividad por la disminución en la radiación lumínica que les hace perder capacidad fotosintética. A causa de la biomasa que producen, son pastos muy extractivos, por lo que mientras más cerca estén del nivel del mar, más exigentes se vuelven en aporte de agua por riego y asimismo, mientras menor potencial fértil tenga el suelo más limitada será su capacidad de producción.

Pennisetum sp. es una gramínea con una alta capacidad de producción de forraje de buena calidad nutricional que, por tratarse de un pasto de corte, permite incrementar la producción por hectárea(Ramírez 2003). Aunque la calidad nutricional del pasto maralfalfa fue descrita de manera reciente por Correa et al. (2002), quienes mostraron que la calidad nutricional cambia con la edad de corte, se hace necesario incrementar la información sobre esta especie, en particular en lo que tiene que ver con el contenido y liberación ruminal de algunos macrominerales.

El pasto imperial es un buen estimulante lactogénico, su calidad nutritiva es media pero su probabilidad y aceptabilidad de uso son altas. El primer corte es a los 3-4 meses y luego los cortes se pueden realizar cada 90-110 días. Se usa al igual que el micay Axonopus micay como diurético en decocción de toda la planta.

\section{MATERIALES Y MÉTODOS}

Área de estudio. El estudio se desarrolló en el Centro Multipropósito Monte las Palmas en la vía que conduce al corregimiento de San José de Purre, situado en el municipio del Atrato, departamento del Chocó, Colombia (Figura 1). La zona se destaca por estar ubicada a una altura de $60 \mathrm{msnm}$, temperatura promedio de $26^{\circ} \mathrm{C}$ y una precipitación de 7250 $\mathrm{mm}$. El sector se caracteriza por ser una zona dedicada en su mayoría a la explotación agrícola y minera con algún aprovechamiento forestal, agropecuario y recreacional, con tierras cultivadas con pan coger y excedentes comercializados en la ciudad de Quibdó a nivel de tiendas (Castro y Barajas 2004).

Métodos. El galpón se caracterizó por ser de estructura de madera, muro de bloque, $\mathrm{H} 40 \mathrm{~cm}$ con refuerzo de madera hasta de $1.80 \mathrm{~m}$, malla metálica para encerramiento, piso de cemento y afirmado, entramado de madera, cubierta de zinc. Se establecieron dos lotes con 400 pollas ponedoras cada uno de la raza Loman brown, en los cuales se realizó el seguimiento de la producción de huevos durante 93 y 99 semanas con el suministro de $100 \mathrm{~g}$ de concentrado ave/día y de los pastos imperial (Axonopus micay, Axonopus scoparius) y maralfalfa (Pennisetum sp.). Se tomaron además registros diarios reflejados en semanas y estos a su vez en períodos que comprenden cuatro semanas.

Se tuvieron en cuenta índices semanales referentes a número de aves promedio, consumo de alimento, promedio de huevos/día, porcentaje de postura y además, se efectuaron observaciones sobre el tipo de alimentación suministrado a las pollas, destacando la actividad que tienen algunas plantas (pasto) en la alimentación animal frente a la convivencia, disminución y abaratamiento del costo que genera el consumo de purina.

\section{RESULTADOSYDISCUSIÓN}

El análisis del registro por período para cada uno de los lotes establecidos aparece por figuras, denotando su interpretación para cada estado de producción.

Lote 1. De la Figura 2 (período 1 al 5) se puede deducir que en este período se presentó un aumento progresivo en la postura de las gallinas, el ascenso se percibe desde la semana 21 en $82 \%$ y se mantuvo la proyección hasta la semana 24 en un $92.5 \%$; de la semana 25 con un $91.4 \%$ a la semana 27 con $89.3 \%$ hay un leve descenso, un punto por semana; a partir de la semana 34 a la 37 hay descenso y recuperación inmediata, repuntando en las semanas 38 y 39 con un $94.5 \%$ y $98 \%$ respectivamente.

A nivel de este período que inicia en la semana 20 y termina en la semana 39 los índices arrojan los datos mostrados en la 
Producción y calidad de huevos. A Castro, et al.

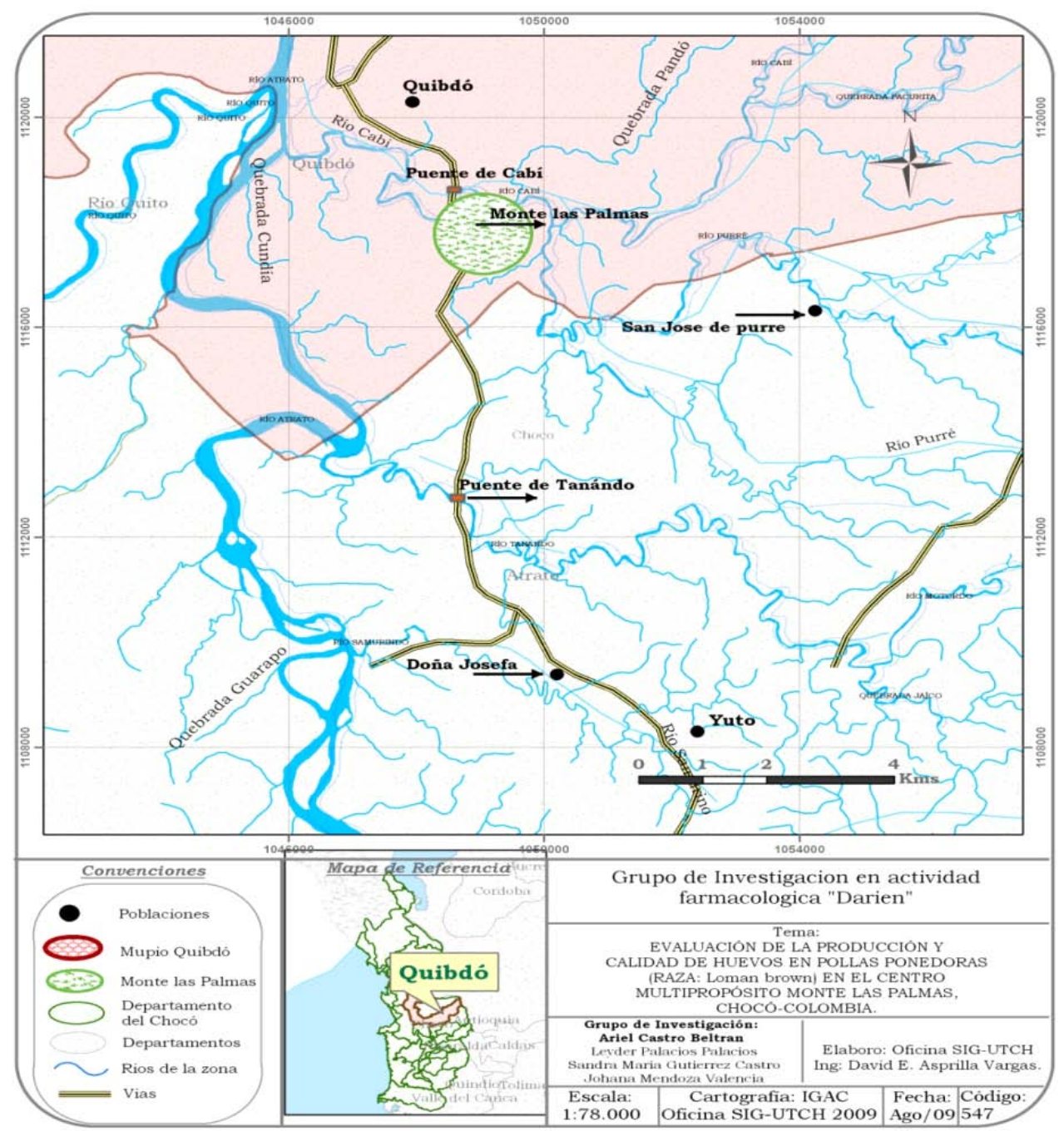

Figura 1. Ubicación del área de estudio.

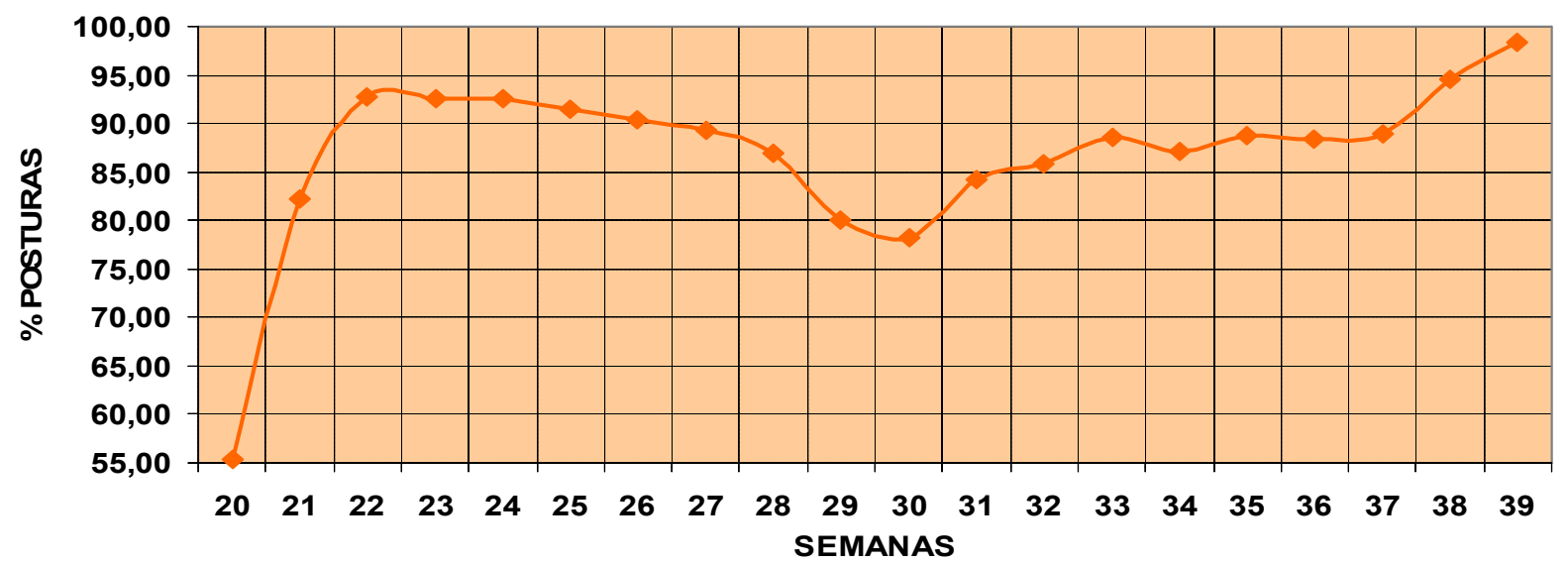

Figura 2. Período comprendido entre la semana 20 y la semana 39. 
Bioetnia Volumen 7 № 1 (enero-junio), 2010

Tabla 1

Índices obtenidos para los períodos 1 al 5

\begin{tabular}{|c|c|c|c|c|c|c|}
\hline \multirow[b]{2}{*}{1 al 5} & \multicolumn{5}{|c|}{ Período } & \multirow[b]{2}{*}{ Final períodc } \\
\hline & 1 & 2 & 3 & 4 & 5 & \\
\hline $\begin{array}{l}\text { Acumulado huevos total desde } \\
\text { iniciación postura }\end{array}$ & $8.023,0$ & $16.862,0$ & $24.544,0$ & $32.527,0$ & $40.834,0$ & 122.790 \\
\hline Promedio de aves & 355,0 & 338,0 & 328,0 & 326,0 & 318,0 & 318 \\
\hline Alimento consumido (gramos) & 994,0 & 975,8 & 949,2 & 916,3 & 902,3 & $4.737,6$ \\
\hline Promedio de huevos/día & $1.146,0$ & $1.263,0$ & $1.097,0$ & $1.140,0$ & $1.187,0$ & 5.833 \\
\hline \multicolumn{7}{|c|}{$\begin{array}{l}\text { Tabla } 2 \\
\text { Índices obtenidos para los períodos } 6 \text { al } 10\end{array}$} \\
\hline & \multicolumn{5}{|c|}{ Período } & \\
\hline 6 al 10 & 6 & 7 & 8 & 9 & 10 & Final período \\
\hline $\begin{array}{l}\text { Acumulado huevos total desde i } \\
\text { niciación postura }\end{array}$ & $49.016,0$ & $56.308,0$ & $62.802,0$ & $68.400,0$ & $74.149,0$ & 310.675 \\
\hline Promedio aves & 299,0 & 291,0 & 287,0 & 276,0 & 271,0 & 271 \\
\hline Alimento consumido (gramos) & 872,9 & 828,8 & 807,8 & 795,9 & 765,1 & $4.070,5$ \\
\hline Promedio de huevos/día & $1.169,0$ & $1.042,0$ & 828,0 & 800,0 & 821,0 & 4.660 \\
\hline
\end{tabular}

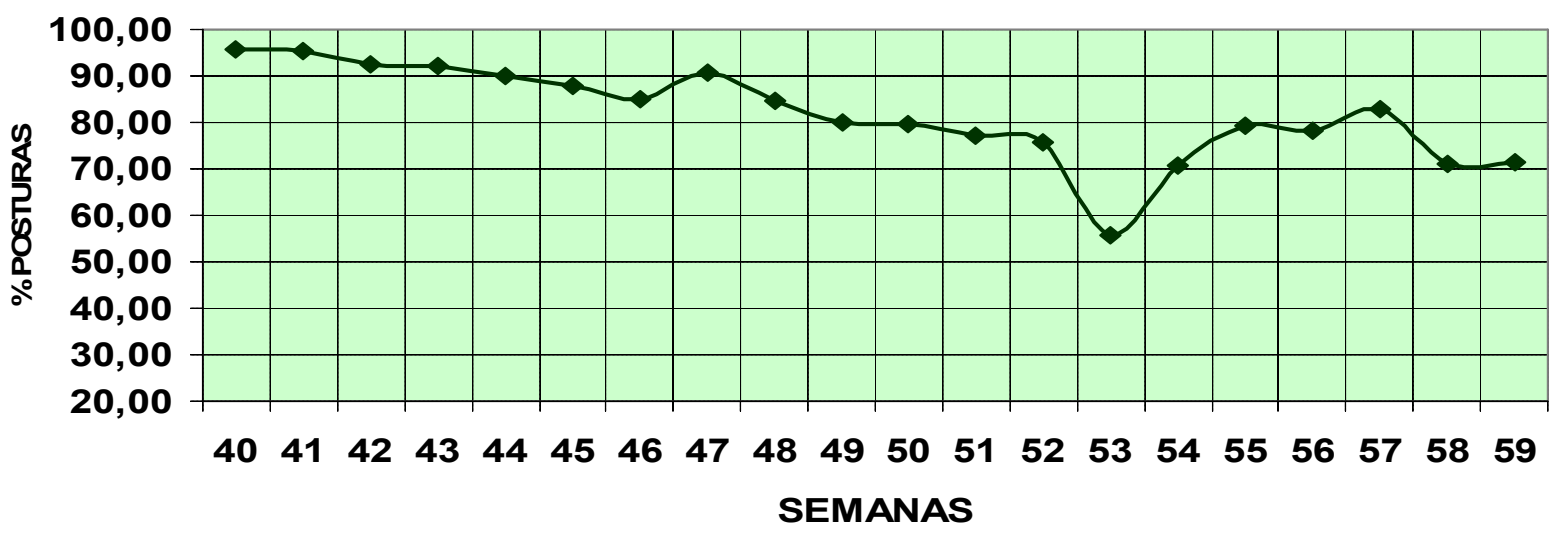

Figura 3. Período comprendido desde la semana 40 a la semana 59

Tabla 1.

La Figura 3 (período 6 al 10) muestra que se detiene la producción de huevos. Empieza a bajar la productividad de los huevos si se tiene en cuenta que en la semana 39 terminó en $98 \%$; se presenta una estabilidad porcentual de $95 \%$ de la producción entre las semanas 40 y 41 , continuó el declive desde la semana 42 con un $92 \%$ hasta la semana 46 en $85 \%$; asciende un poco hasta $90.7 \%$ en la semana 47 ; vuelve a bajar la productividad a partir de la semana 48 en un $84 \%$ hasta la semana 53 en $55.8 \%$; se da un proceso de recuperación en las semanas 54 y 55 , cuando se ubica en un porcentaje aproximado de $70 \%$ y $79 \%$, tres puntos y medio; mientras que para la semana 56 baja la productividad a $77.5 \%$, y culmina con un $72 \%$. Durante este período se obtuvieron los índices de producción mostrados en la Tabla 2.

Al observar la Figura 4 (período 11 al 15) se puede concluir que hubo una estabilidad en las primeras siete semanas conservando la producción de las cinco últimas semanas del período anterior; se parte de una producción de huevos de $73.5 \%$ y en la semana 60 se puede apreciar que la productivi- 


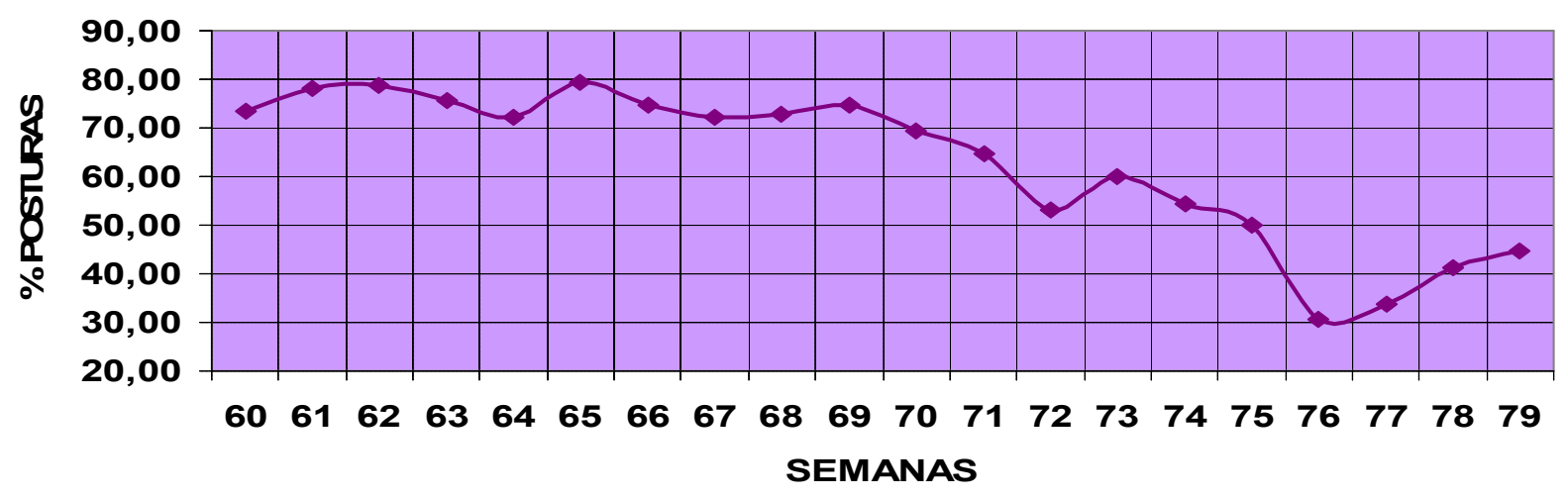

Figura 4. Período comprendido desde la semana 60 a la semana 79.

Tabla 3

Índices obtenidos durante los períodos 11 al 15

\begin{tabular}{lcccccc}
\hline $\mathbf{1 1}$ al 15 & $\mathbf{1 1}$ & $\mathbf{1 2}$ & $\begin{array}{c}\text { Período } \\
\mathbf{1 3}\end{array}$ & $\mathbf{1 4}$ & $\mathbf{1 5}$ & Final período \\
\hline $\begin{array}{l}\text { Acumulado huevos total desde } \\
\text { iniciación postura }\end{array}$ & $79.885,0$ & $85.461,0$ & $90.651,0$ & $94.543,0$ & $97.179,0$ & $\mathbf{4 4 7 . 7 1 9}$ \\
$\begin{array}{l}\text { Promedio aves } \\
\text { Alimento consumido (gramos) }\end{array}$ & 271,0 & 268,0 & 266,0 & 260,0 & 247,0 & $\mathbf{2 4 7}$ \\
\begin{tabular}{l} 
Promedio de huevos/día \\
\hline
\end{tabular} & 858,8 & 755,3 & 749,7 & 737,1 & 702,8 & $\mathbf{3 . 7 0 3 , 7}$ \\
\hline
\end{tabular}

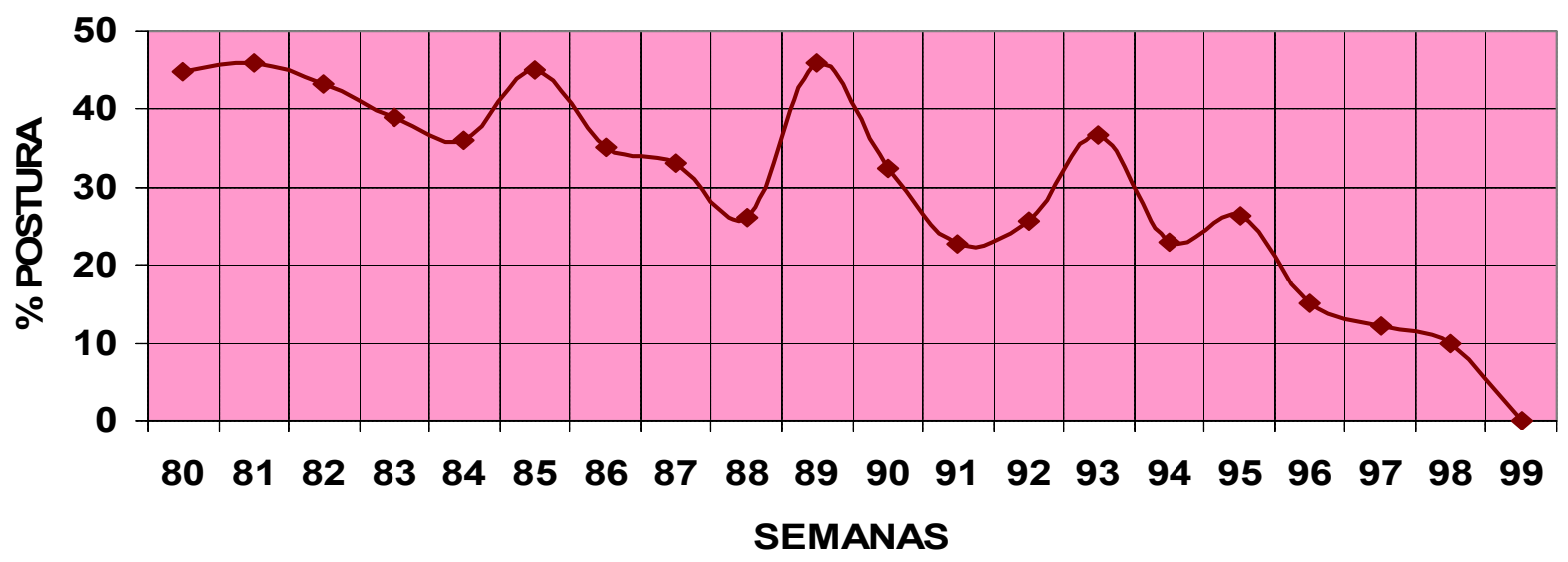

Figura 5. Período comprendido entre la semana 80 a la 99.

dad baja y se recupera de una semana a otra, conservando una estabilidad entre $70 \%$ y $75 \%$ hasta llegar a la semana 70 con $69 \%$ donde se pierde la estabilidad que desciende de manera vertiginosa hasta ubicarse en la semana 72 con un 53\%; en las semanas siguientes se presenta disminución en la productividad que llega hasta $44.7 \%$ en la semana 79 final del período. Es importante señalar la progresión matemática que se da de una semana a otra, destacándose la baja productividad y leve recuperación en este período.
Los índices en este período presentan una disminución considerable en comparación con los anteriores (Tabla 3).

En el período comprendido entre las semanas 16 a la 20 (Figura 5), continúa la baja productividad de los huevos, de forma irrecuperable; se parte de una productividad aproximada de $45 \%$ en la semana 80 , se presenta un leve aumento en la semana 81 que representa un crecimiento de un punto porcentual con $46 \%$, pero a partir de la semana 82 con un $43 \%$ se empieza a evidenciar que declina la producción de huevos 
Bioetnia Volumen 7 № 1 (enero-junio), 2010

Tabla 4

Índices obtenidos de los períodos 16 al 20

\begin{tabular}{|c|c|c|c|c|c|c|}
\hline \multirow[b]{2}{*}{16 al 20} & \multicolumn{5}{|c|}{ Período } & \multirow[b]{2}{*}{ Final período } \\
\hline & 16 & 17 & 18 & 19 & 20 & \\
\hline $\begin{array}{l}\text { Acumulado huevos total desde } \\
\text { iniciación postura }\end{array}$ & $100.163,0$ & $102.571,0$ & $104.239,0$ & $105.102,0$ & $105.287,0$ & 517.362 \\
\hline Promedio aves & 246,0 & 246,0 & 140,0 & 71,0 & 71,0 & 71 \\
\hline Alimento consumido (gramos) & 690,2 & 688,8 & 574,0 & 342,7 & 149,1 & $2.444,8$ \\
\hline Promedio de huevos/día & 426,0 & 344,0 & 238,0 & 123,0 & 26,0 & 1.157 \\
\hline
\end{tabular}

Tabla 5

Índices obtenidos en las semanas 18 a la 37 en el Lote 2

\begin{tabular}{lcccccc}
\hline \multirow{1}{*}{$\mathbf{1}$ al 5 } & \multicolumn{5}{c}{ Período } & Final período \\
\cline { 2 - 6 } & $\mathbf{1}$ & $\mathbf{2}$ & $\mathbf{3}$ & $\mathbf{4}$ & $\mathbf{5}$ & Fina \\
\hline $\begin{array}{l}\text { Acumulado huevos total desde } \\
\text { iniciación postura }\end{array}$ & $4.316,0$ & $14.493,0$ & $24.255,0$ & $33.948,0$ & $42.804,0$ & $\mathbf{1 1 9 . 8 1 6}$ \\
$\begin{array}{l}\text { Promedio aves } \\
\text { Alimento consumido (gramos) }\end{array}$ & 402,0 & 399,0 & 376,0 & 368,0 & 357,0 & $\mathbf{3 5 7}$ \\
Promedio de huevos/día & 6125,6 & $1.224,2$ & $1.109,5$ & $1.038,8$ & $1.015,7$ & $\mathbf{5 . 5 1 3 , 8}$ \\
\hline
\end{tabular}

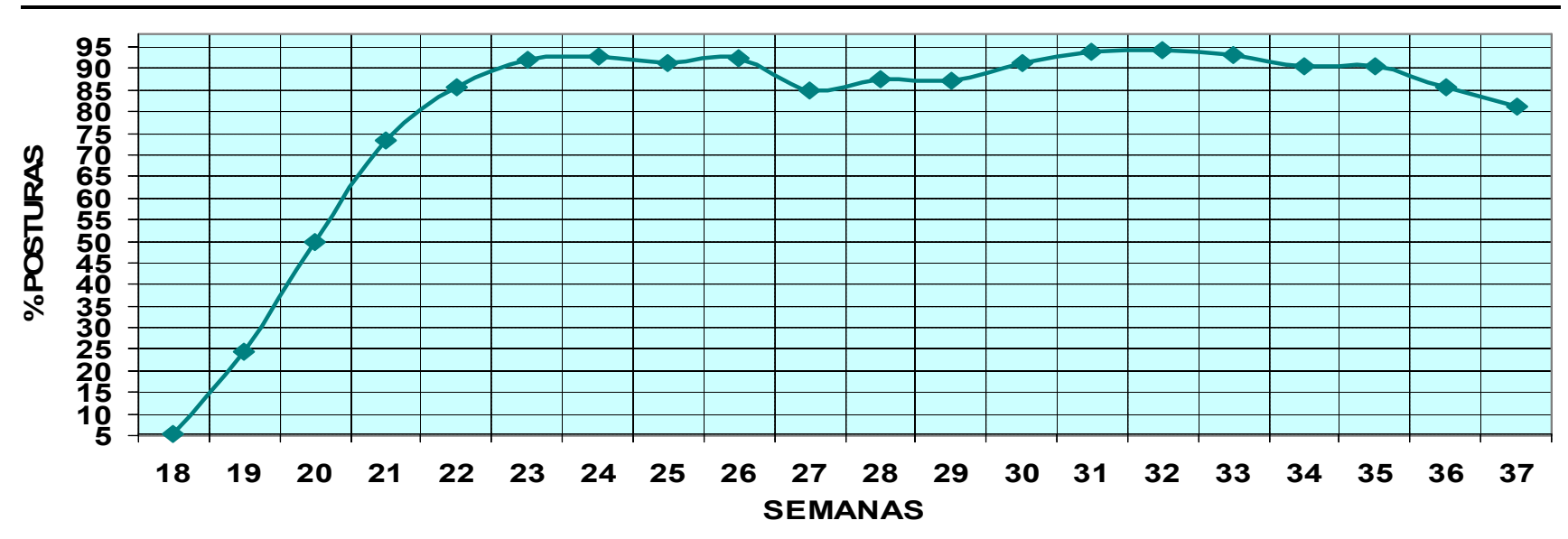

Figura 6. Período comprendido desde la semana 18 a la semana 37 (Lote 2).

y se presentan picos de recuperación de hasta veinte (20) puntos a corto plazo que no logran superar los descensos en la producción lo que indica que el proceso de producción de huevos llega a su final; esto se hace evidente en la semana 99.

En general la producción durante las semanas 79 y la 99 , es baja y no presenta mayores incrementos, los índices son inferiores a los obtenidos en períodos anteriores, situación que marca el final del ciclo productivo (Tabla 4).

Lote 2. La Figura 6 permite discurrir que en este período se presentó aumento progresivo en la postura de las gallinas: en la semana 18 se inicia con una producción de huevos de $12.7 \%$ y se mantiene una proyección de aumento de $92.9 \%$ en la semana 24 . Se observa que estos altos niveles de productividad se mantienen estables hasta la semana 35, donde empieza a evidenciarse que disminuye en forma leve la productividad hasta alcanzar un $81.1 \%$ al final del período en la semana 37. A nivel de este período que inicia en la semana 18 y termina en la semana 37 los índices arrojaron los resultados mostrados en la Tabla 5.

Al observar la Figura 7, se puede inferir que para la semana 
Producción y calidad de huevos. A Castro, et al.

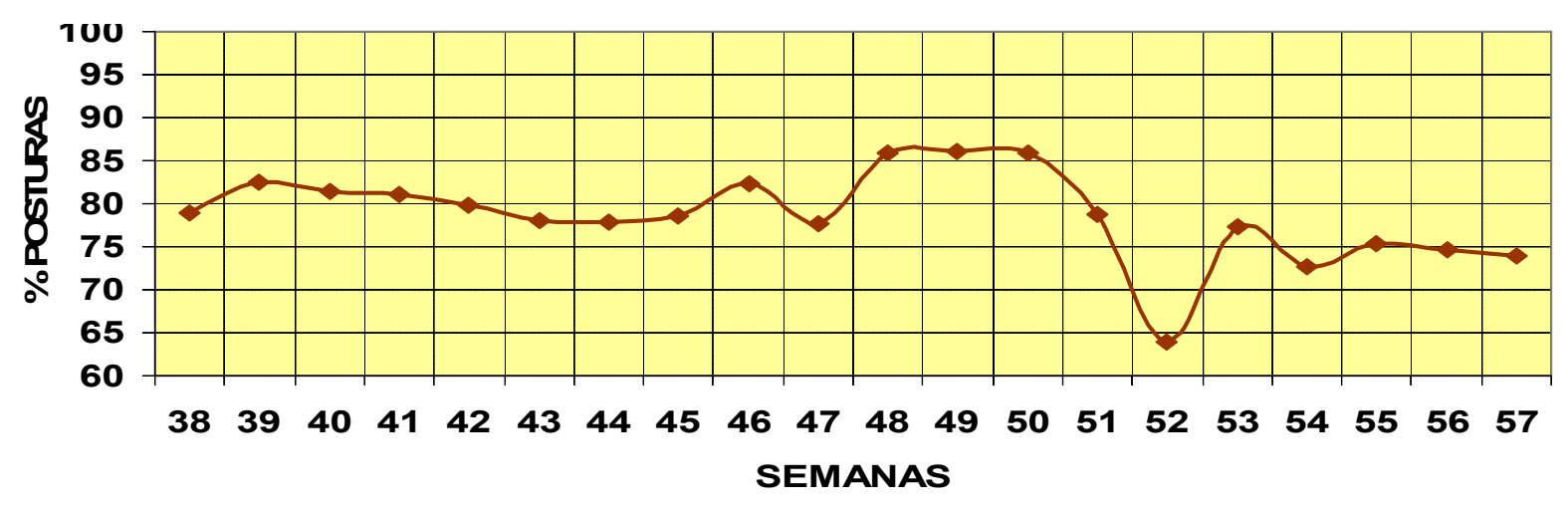

Figura 7. Período comprendido entre la semana 38 y la semana 57 (Lote 2).

Tabla 6

Índices obtenidos en los períodos del 6 al 10 del Lote 2

\begin{tabular}{lcccccc}
\hline \multirow{1}{*}{$\mathbf{6}$ al 10 } & $\mathbf{6}$ & $\mathbf{7}$ & $\mathbf{8}$ & $\mathbf{9}$ & $\mathbf{1 0}$ & Final período \\
\cline { 2 - 6 } & $\mathbf{6}$ & $58.301,0$ & $66.075,0$ & $73.110,0$ & $79.667,0$ & $\mathbf{3 2 7 . 9 1 9}$ \\
\hline $\begin{array}{l}\text { Acumulado huevos total desde } \\
\text { iniciación postura }\end{array}$ & $50.766,0$ & & & & & \\
$\begin{array}{l}\text { Promedio aves } \\
\text { Alimento consumido (gramos) }\end{array}$ & 351,0 & 336,0 & 334,0 & 325,0 & 314,0 & $\mathbf{3 1 4}$ \\
Promedio de huevos/día & 991,9 & 959,0 & 936,6 & 929,6 & 895,3 & $\mathbf{4 . 7 1 2 , 4}$ \\
\hline
\end{tabular}

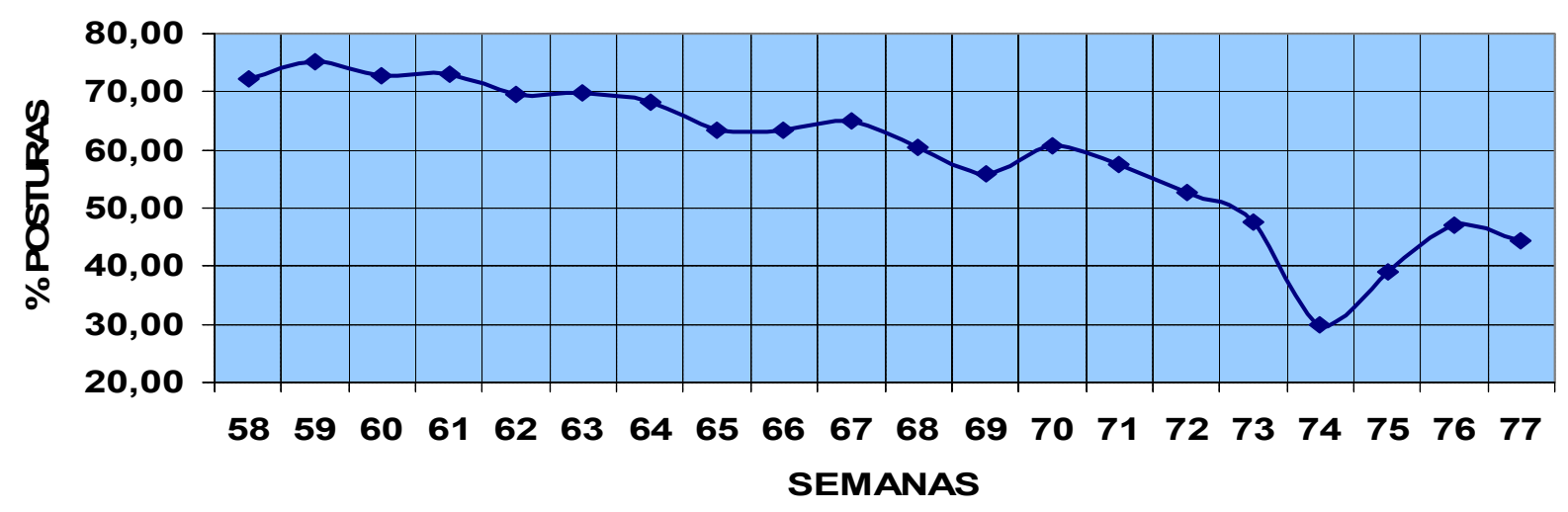

Figura 8. Período comprendido entre la semana 58 a la semana 77 (Lote 2).

38 se inicia un proceso de disminución en la postura de las gallinas, pues se presenta una producción de $78.9 \%$, un punto menos con respecto a la última semana del período anterior; para la semana 39 la producción se ubicó en $82.6 \%$, que permanece más o menos estable hasta la semana 51 con un promedio que varió entre $77 \%$ y $86 \%$. Durante la semana 52 se presentó el mayor descenso con un $63.9 \%$ de productividad, lo que se puede relacionar con el desabastecimiento de concentrado en la ciudad de Quibdó, ocasionado por un paro armado en las vías de acceso. Para la semana 53 hubo una notable mejoría en la productividad de las gallinas ubicándose en un $77.4 \%$, se presentaron bajas en la producción y recuperación inmediata a partir de la semana 54 y 55 para estabilizarse en la semana 57 con un porcentaje de $73.9 \%$. Los índices de producción obtenidos durante este período se presentan en la Tabla 6.

De acuerdo con la Figura 8, la semana 58 inicia con un porcentaje de $72.2 \%$ de producción, que se mantiene en promedio estable hasta la semana 62 , donde se inicia un descenso que alcanza el $44.4 \%$ en la semana 77 . 
Tabla 7

Índices obtenidos en los períodos del 11 al 15 del Lote 2

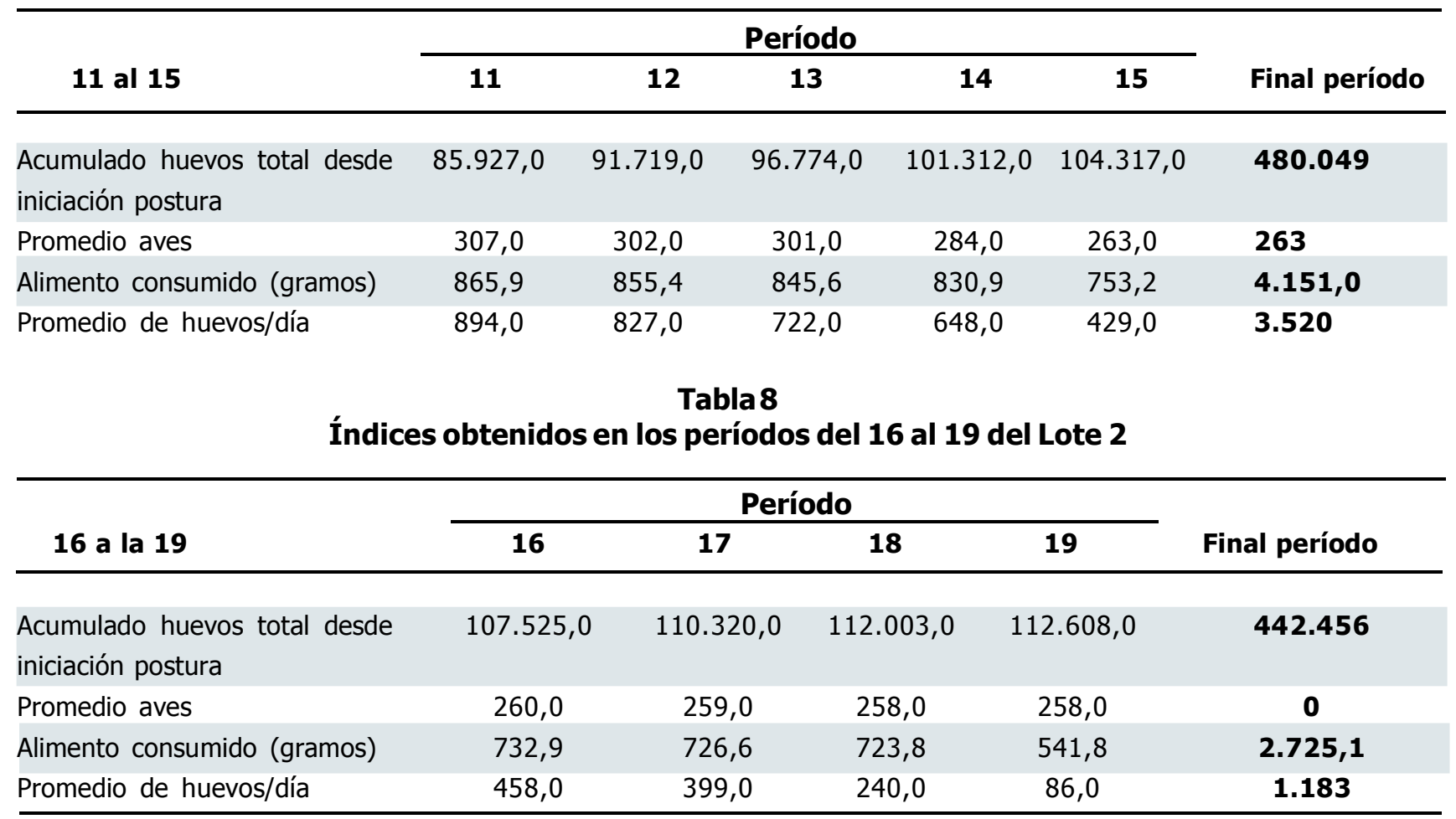

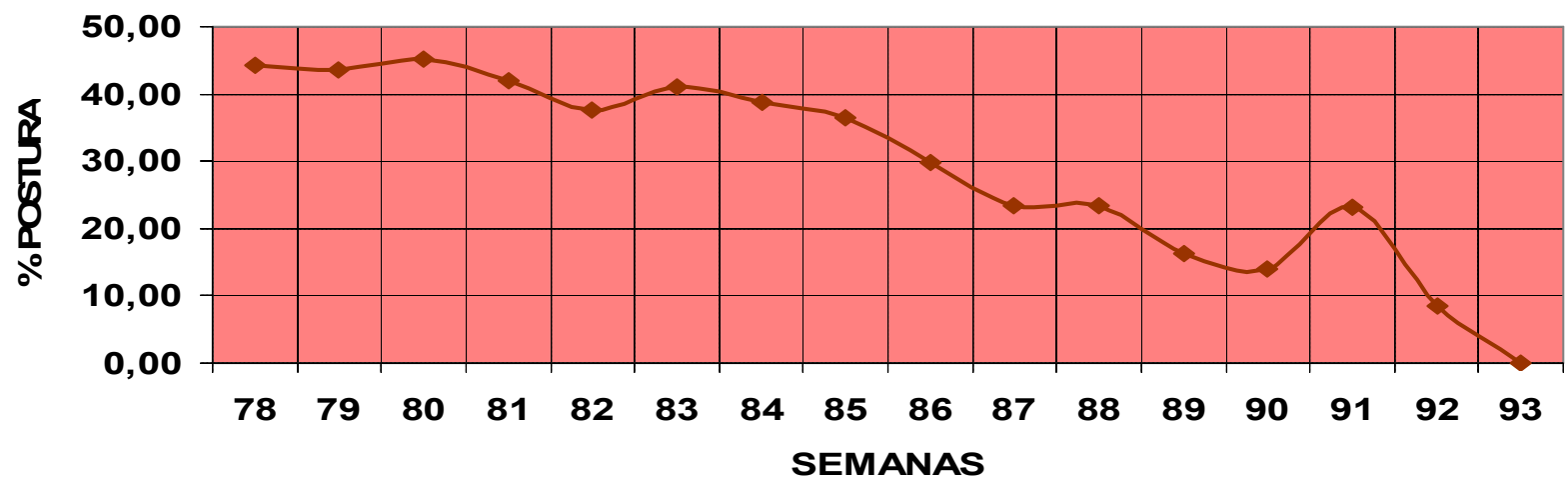

Figura 9. Período comprendido desde la semana 78 a la semana 93 (Lote 2).

Los índices de producción de este período muestran una estabilidad durante la mayor parte del tiempo y una disminución hacia el final, indicando que la producción de huevos comienza su etapa final (Tabla 7).

El período comprendido entre las semanas 78 y 93, está marcado por la estabilidad de las producciones más bajas del Lote 2 (Figura 9). En la semana 78 se informa un $44.2 \%$. A partir de la siguiente semana la producción de huevos desciende de manera constante con algunos leves aumentos que no aportan estabilidad a la curva y que presentan un descenso vertiginoso que en la semana 90 alcanza un $14 \%$. Aunque la productividad se recupera de forma muy leve hasta un $23.1 \%$, en la semana 93 sufre una fuerte caída a 8.5\%, lo que indicó el final del proceso e indujo al sacrificio de las aves en este período. En este período que inicia en la semana 78 y termina en la semana 93 se obtuvieron los índices mostrados en la Tabla 8.

\section{LACALIDAD DEL HUEVO PRODUCIDO}

La calidad de los huevos se determinó por el tipo de alimentación suministrada a las pollas, que se destacó sobre todo por la utilización de los pastos imperial (Axonopus micay) y maralfalfa (Pennisetum $\mathrm{sp}$.) (las cantidades suminis- 
tradas no se pesaron). Estas variables influyeron de manera significativa en la convivencia, pues se observó menos canibalismo; también se presentó un mejoramiento en la textura externa del huevo y la calidad de la yema con tonalidades de amarillo intenso, además fue muy frecuente la aparición de huevos con dos yemas e incluso con tres yemas y con la clara muy diferenciada en cuanto a su aspecto físico.

Según las observaciones efectuadas en el Lote 1, en el período 1 los huevos fueron de menor tamaño, pero el tamaño y la cantidad fue aumentando de forma progresiva a través de las semanas; en el período 2, se evidenció el aumento significativo del consumo de los pastos y mejoramiento de la calidad del huevo y la presencia de brotes de canibalismo; para el período 3, la frecuencia de consumo de pasto aumentó y con ello mejoró de manera sustancial el tamaño de los huevos, dándose la aparición de huevos con dos yemas y en las dos últimas semanas se presentaron pequeños brotes de canibalismo. En el período 4, se denotaron huevos grandes muy uniformes de color externo café acentuado, comenzaron a parecer los huevos de tres yemas; en los períodos 5 y 6 , se encontró que la calidad y el tamaño del huevo se mantuvo, aunque hubo que desechar algunas gallinas que se encontraban en mal estado; en el período 7, continuó estable la calidad del huevo, se aumentó la cantidad de los mismos y el suministro del pasto; para el período 8, la calidad se mantuvo en cuanto al tamaño del huevo y su aspecto físico así como el consumo eficiente del pasto; en el período 9 , hubo aumento en el consumo de pasto y la calidad del huevo se mantuvo; en los períodos $10,11,12,13$ y 14, se mantuvieron los estándares de consumo de pasto y la calidad del huevo; pero en el período 15, aunque la calidad y el aspecto del huevo se mantuvo se presentó muerte de pollas por picaduras entre ellas; en los períodos 16 y 17, se mantiene el tamaño y color de los huevos, el consumo de los pastos sigue siendo bueno y en los períodos 18,19 y 20 , los aspectos antes mencionados de la calidad del huevo se mantuvieron iguales, aunque por la baja productividad de las pollas ponedoras fueron sacrificadas.

Al analizar el comportamiento general del Lote 2 se encuentra que en el período 1 , los huevos fueron de menor tamaño, pero aumentaron de tamaño rápidamente al final del período y mejoró su aspecto físico; en el período 2 , se evidenció aumento significativo del consumo de los pastos por parte de las aves y mejoramiento en el tamaño del huevo; para el período 3 , aunque se presentaron brotes de canibalismo en las dos últimas semanas, el tamaño del huevo mejoró de manera sustancial. En el período 4, siguió el aumento del consumo de pasto y al final del período se observaron huevos muy grandes con dos y tres yemas; en el período 5 , el consumo de los pastos fue más evidente, porque se aumentó la cantidad de forma progresiva; para el período 6 , la calidad del huevo permaneció constante y en general fue alta; en los períodos
7, 8, 9 y 10, continuó estable la calidad del huevo en sus aspectos físicos como el color, tamaño, textura de la cáscara y la yema presentó color amarillo intenso; además se presentó estabilidad en el consumo de pasto. En los períodos 11, 12, 13 y 14 , el consumo de pasto fue considerable y la calidad del huevo excelente; en el período 15 , aunque se descartaron algunas aves por encontrarse en mal estado no hubo variación ni en la calidad ni en el consumo de pasto; en los períodos 16,17 y 18, el consumo de los pastos continuó siendo muy bueno al igual que el aspecto físico del huevo (color, tamaño y forma); $y$ en el período 19, la calidad del huevo se mantuvo, lo mismo que el consumo del pasto, aunque se sacrificaron las gallinas por la baja producción de huevos.

\section{CONSIDERACIONES FINALES}

Es bueno resaltar que la productividad máxima de huevos en las pollas ponedoras se dio entre el sexto (porcentaje de postura alcanza al $93.90 \%$ ) y cuarto (porcentaje de postura alcanza al 93.2\%) período respectivamente en los dos lotes investigados; aunque en los períodos subsiguientes, se presentaron algunos picos. Sin embargo, se observa el descenso vertiginoso en la productividad de huevos de las pollas ponedoras en el período número 15 de ambos lotes evaluados, como se observa en los Anexos 1 y 2.

En los primeros períodos los huevos eran pequeños, la textura de la cáscara al igual que el color no estaban bien definidos, con el tiempo y el control de la alimentación mejoró el aspecto físico de los huevos, resaltando el tamaño (grande) y el color bien diferenciado; lo que contrastó con el alto consumo de pastos por parte de las aves que cada vez más eran exigentes, teniendo como consecuencia:

a. La generación de huevos de gran tamaño, color bien acentuado y la aparición de dos y tres yemas;

b. El aumento del consumo de pasto, por lo que fue necesario aumentar los puestos de abastecimiento de los mismos, disminuyendo la muerte por canibalismo, genéticamente determinada en este tipo de aves.

\section{LITERATURA CITADA}

Betancur, J. F. 2004. Comparación de dos procedimientos matemáticos para estimar la degradabilidad efectiva en rumen. Medellín: Universidad Nacional de Colombia; 128 pp.

Bernal Restrepo, J. I., Moreno Durán, G. 1997. Pastos para corte y pastoreo. Medellín: Editorial de la Biblioteca Universidad Pontificia Bolivariana

Bonino, M. 1994. El pollo y huevo campero. Med. Vet. Zulma Canet. Public. del Instituto Nacional de Tecnología Agropecuaria INTA. Villa María, Córdoba, Argentina, 58 p. [fecha de acceso 23 de octubre 2008]. URL disponible en: http://www.inta.gov.ar/pergamino/investiga/grupos/avicultura/publi_ave.htm

Castro, B. A., Barajas, P. A. 2004. Avalúo comercial de un inmueble. Atrato: Editorial Temis; $67 \mathrm{pp}$.

Correa, H. J., Arroyave, H., Henao, Y., López A., Cerón, J. 2002. Maralfalfa. Mitos y realidades. Despertar lechero. 22 (1): 79-88. 


\section{Bioetnia Volumen 7 № 1 (enero-junio), 2010}

Anexo 1

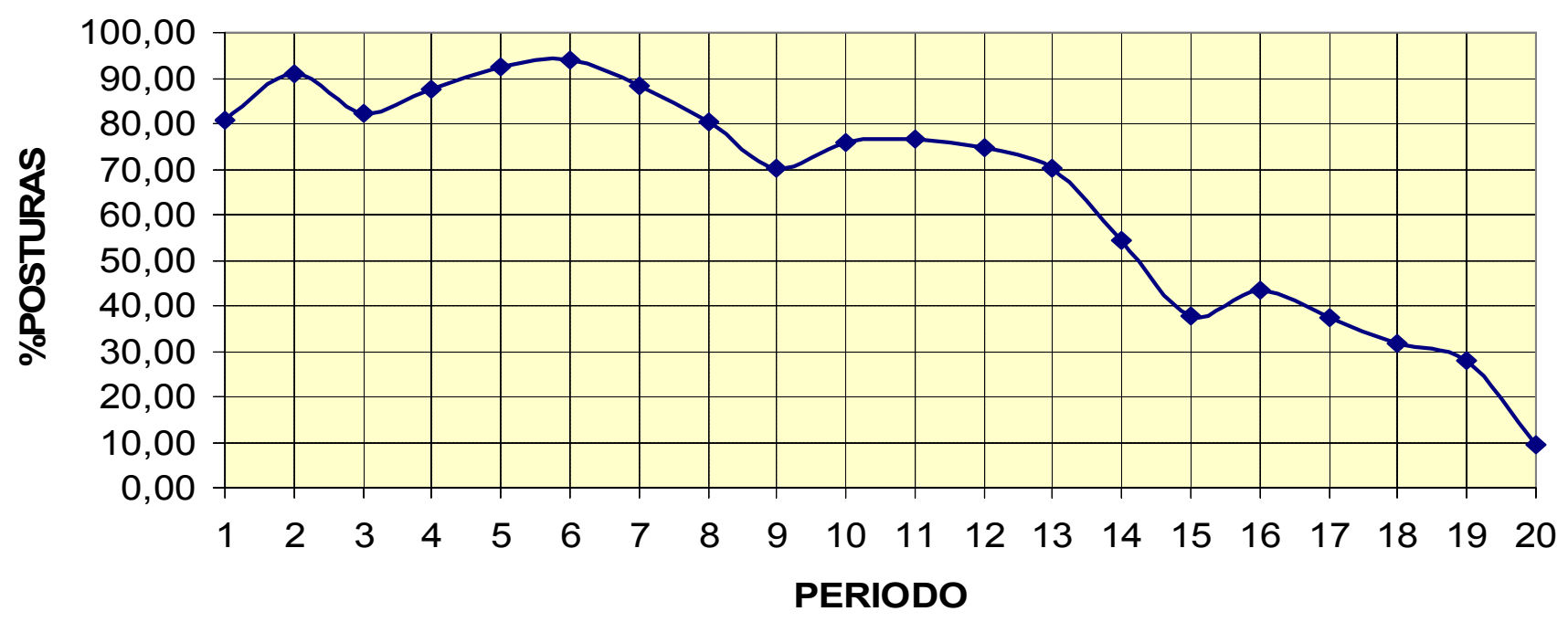

Anexo 2

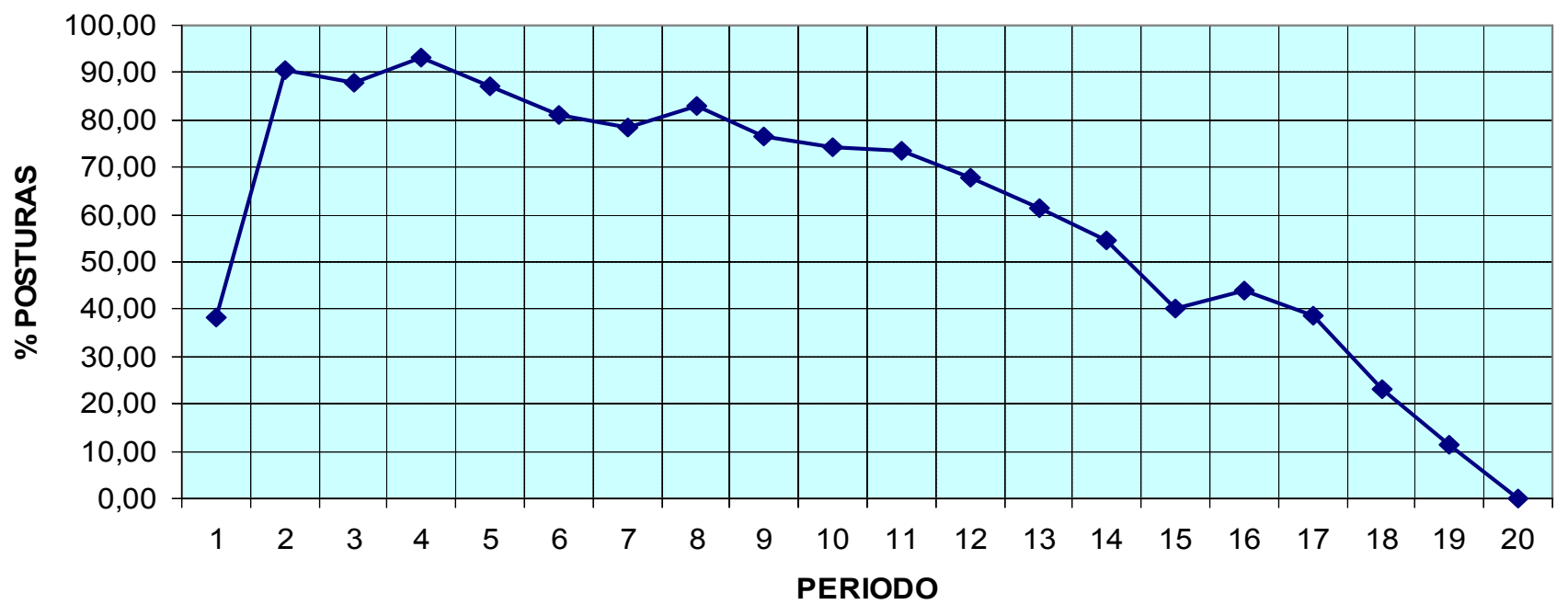

Ramírez, G. 2003. Pasto maralfalfa, un manjar para hatos ganaderos. El Colombiano. Medellín. Sábado 16 de agosto, 4b.

Romero, J. 2007. Campo Experimental «Valle de Culiacan» km. Culiacán:
Centro de Validación y Transferencia de Tecnología de Sinaloa (CVTTS) río Fuerte. 38 pp.

Rua, F. 2008. Pastos de corte para el trópico. Medellín: Universidad de Antioquia; $62 \mathrm{pp}$. 